\title{
Location-Aware and Personalized Collaborative Filtering for Web Service Recommendation : A Review
}

\author{
Chinnu Priya J.V. \\ PG scholar \\ Department of Computer Science \\ College of Engineering Perumon,Kerala, India
}

\author{
Suja Rani M.S. \\ Assistant Professor \\ Department of Information Technology \\ College Of Engineering Perumon, Kerala, India
}

\begin{abstract}
As the number of web services with similar functionality increases, the service users usually depend on web recommendation systems.Now a days the service users pay more importance on non functional properties which are also known as Quality of Service(QoS) while finding and selecting appropriate web services.Collaborative filtering[3] approach predicts the QoS values of the web services effectively.Existing recommendation systems rarely consider the personalized influence of the users and services in determining the similarity between users and services. The proposed system is a ranking oriented hybrid approach which integrates user-based and item-based QoS predictions.Many of the non-functional properties depends on the user and the service location. The system thus employs the location information of users and services in selecting similar neighbors for the target user and service and thereby making personalized service recommendation for service users.
\end{abstract}

\section{General Terms}

Service computing, Recommendation

\section{Keywords}

Web services, Collaborative filtering, Location-aware, QoS prediction, Service recommendation

\section{INTRODUCTION}

Web service has been emerged as a promising technique to support inter-operable machine-to-machine interaction which provides a method of communication between electronic devices over a network.As the number of web services with similar functionality has increased rapidly over the internet the web service discovery is not a challenging task but selection and recommendation are becoming more important.Optimality of a web service depends on its performance and performance is measured through Quality of Service i.e. QoS.QoS is the set of non functional properties[7] of a web service which includes response time,price,failure rate and so on.

Recommendation system initially searches for the list of web services those having similar functionality, which the user requested and finally the optimal web services are recommended to users.Collaborative filtering is widely employed in web service rec-
ommendation.Existing QoS prediction[7],[10] methods rarely finds the similarity of users,services and location of users into consideration.The proposed method uses both the location of users and web services on selecting similar neighbors[8] for the target user or service.

\section{RECOMMENDATION SYSTEM}

\subsection{QoS based service ranking}

QoS based service ranking and their selection for a best web service are initially based on the calculation of satisfaction scores[1] of each web service.Basic steps includes: the registration of web services in a service directory(SD)[1], users specifying the QoS requirements to the service directory.The SD initially matches user's functional requirements followed by the calculation of satisfaction scores for each service.The SD lists out the services based on the satisfaction scores[1].

QoS-based service ranking and selection method helps users to select the services that best satisfy their QoS requirements. The top ranked service with the largest satisfaction score is the service that best satisfy user's QoS requirements.

\subsection{Collaborative filtering}

Collaborative filtering[3] is most commonly used in present web service recommendation systems. The basic idea in CF is to make automatic predictions about the interests of a user by gathering preferences or taste information from many users. The processing in $\mathrm{CF}$ algorithms are based on user-item matrix.CF techniques are generally divided into two broad categories- model based[6],[2] and memory based[3][2].

2.2.1 Model based collaborative filtering. This method first develop a model of user ratings and provides item recommendation.Probabilistic approaches are being used in this method and model building process is done with the machine learning algorithms like Bayesian network,rule based and clustering approaches.

2.2.2 Memory based collaborative filtering. Memory based collaborative filtering is also known as neighborhood-based CF and it makes use of the entire user-item database to generate a prediction.Based on the user neighborhood or item neighborhood neighborhood-based CF can be further classified into:

(1) User-based CF 
(2) Item-based CF

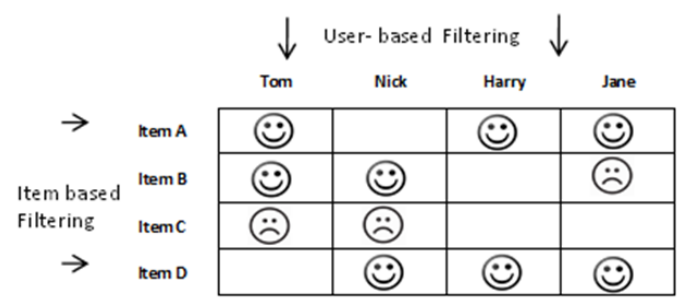

Fig. 1. Compare user-based and item-based filtering

User Based filtering can be explained as: In fig 1, it is found that users Tom and Nick seem to have similar interests as they both liked item B and both dislikes item C.So the item A will be recommended to Nick.Similarly item-based filtering[2] can be explained as : Considering an item D,two users who liked item D Harry and Jane also liked another item A.From this observation we can conclude that people who liked item D will also like item A.So item A will be recommended to Dick.

User-based CF provides a subset of appropriate users as neighbors based on their similarities to the active user and the Item-based $\mathrm{CF}$ will retrieve those items similar to the ones the active user preferred in the past.

\subsection{Similarity Calculation}

Pearson Correlation Coefficient $(P C C)$. It is used to measure user similarity[2] in recommendation systems. It measures the similarity between two service users based on the QoS values of Web services.PCC[3] similarity $\operatorname{sim}(a, b)$ of two service users ranges from -1 to 1 .Two service users have similar Web service usage experiences if the PCC value is positive and a negative PCC value indicates that their experiences are opposite. The value is null when two service users have no commonly invoked web service.

\begin{tabular}{lllll}
\hline & $I_{1}$ & $I_{2}$ & $I_{3}$ & $I_{4}$ \\
\hline$U_{1}$ & 4 & $?$ & 5 & 5 \\
$U_{2}$ & 4 & 2 & 1 & \\
$U_{3}$ & 3 & & 2 & 4 \\
$U_{4}$ & 4 & 4 & & \\
$U_{5}$ & 2 & 1 & 3 & 5
\end{tabular}

Fig. 2. A simple rating matrix

After calculating the similarity[3], a set of similar neighbors[8] can be found which is considered to be one the important stem in CF.The neighbor selection algorithms selects $\mathrm{K}$ users as his neighbor that are most similar to an active user.Similarly, these algorithms can also find $\mathrm{K}$ Web services that are most similar to a target Web service.

\subsection{Integrating Locations of Users and Services into Similar Neighbor Selection}

Location information of both service users and Web services[5] can be found easily.As the IP address of the user is known it is much easier to locate AS number and the country where he is located.Similarly the location of web services are also found.The location information are then processed to produce a set of similar users and similar services that are closed to each other.

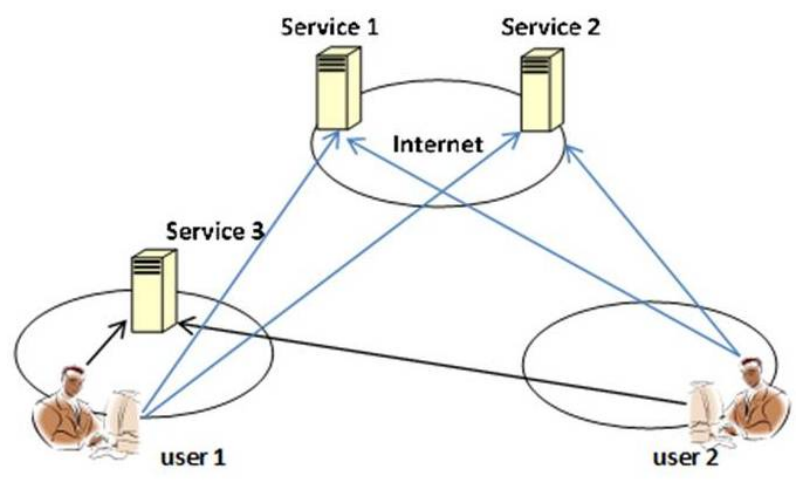

Fig. 3. Influence of user location

The network performance is likely to be poor when the service user and the service are located at different networks. The low performance are mainly due to the transfer delay and the limited bandwidth of the networks.Performance are found to be high if the user and the service are located in the same network.In figure 3 , user 1 and 2 are located at two different geographical regions that are far from each other.Both user 1 and user 2 will experience a similar QoS values on accessing service 1 and 2 and they are similar.Service 3 is found to be in the same network of user 1 and therefore it is closer to him and the service is far away from user 2 and the QoS both users will obtain a different QoS values with respect to service 3 .

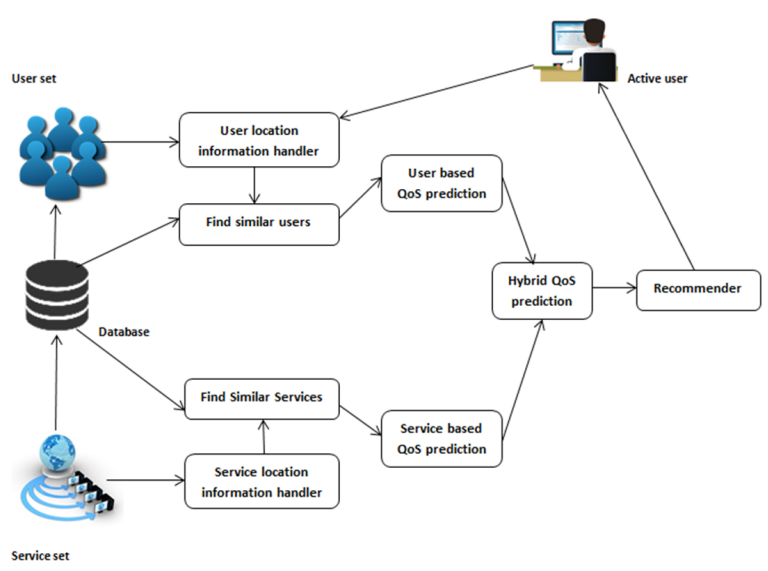

Fig. 4. Recommendation System 
The recommendation system can be explained when an active service user searches for high-quality Web services in a Web service discovery system or the system is recommending high-quality Web services to an active user.The process is done by predicting the QoS values for the web services. The recommendation system as a whole can be explained using the Fig 4 .

The system consists of :

(1) User location information handler : This is used to find the location information of a user which includes the AS number and the country name according to the user's IP address.

(2) Service location information handler :This is used to find the location information of Web services that includes the AS number and the country in which the Web Services are located.

(3) Find similar users: This module finds similar service users who are similar to the active user by considering both the user's QoS values and their corresponding locations.Similarity computation is done with a weighted Pearson Correlation Coefficient.

(4) Find similar services: This module finds similar web services for a target web service by considering both the users QoS values and their corresponding locations. Similarity computation is done with a weighted Pearson Correlation Coefficient.

(5) User-based QoS prediction : After finding similar users from the above step, QoS values are predicted accordingly for an active user.

(6) Service-based QoS prediction : After finding similar services from the above methods, $\mathrm{QoS}$ values are predicted.

(7) Hybrid QoS prediction:The final QoS predictions are obtained by combining the user based QoS prediction and the servicebased QoS prediction.

(8) Recommender:After predicting QoS values, recommendation ofWeb services are given to the active user.

\section{COMPARISON WITH THE OTHER RECOMMENDATION SYSTEMS}

(1) Existing approaches fail to recognize the QoS variation with change in the physical locations of users and services[5].

(2) Current web service recommendation systems provide a recommendation result with no transparency into the reason behind the recommendation results.

(3) Model based methods cannot successfully handle the dynamic user-service matrix.Matrix factorization(MF)[6],[9] is used in this method for QoS prediction.MF model has to be recomputed when there is a change in the matrix.

(4) The recommendation systems are generally based on User-based QoS prediction or item-based QoS prediction.Integrating both the methods for QoS predictions will provide a better recommendation than the individual predictions.

\section{CONCLUSION}

Collaborative filtering techniques presents a QoS based recommendation system.Basically the system will predict the QoS value of a web service and will recommend the best for an active user.This can be integrated with the location information of both users and the services on finding the similar neighborhood results in better QoS predictions.As the IP address of the user is known it is much easier to locate AS number and the country where he is located. Similarly the locations of web services are also found. The location information is then processed to produce a set of similar users and similar services that are close to each other.Comparing with other recommendation systems and the existing methods rarely consider the user and service location in determining QoS values.The existing recommendation systems are either based on user-based or item-based QoS prediction. Integrating both the methods for QoS predictions along with the location information will provide a better recommendation than the individual predictions. The system can be improved by integrating different non functional properties into consideration which helps in providing a better results. 27

\section{REFERENCES}

[1] S. S. Yau, Y. Yin,"QoS-based service ranking and selection for service based systems", in Proc. of the International conference on Services Computing, Washington DC, USA, July, 2011, pp. 56 - 63.

[2] B. Sarwar, G. Karypis, J. Konstan, and J. Riedl, "ItemBased Collaborative Filtering Recommendation Algorithms", in Proc.10th Intl Conf. World Wide Web, 2001, pp. 285-295.

[3] ]Z. Zheng, H. Ma, M. R. Lyu, and I. King "QoS-Aware Web Service Recommendation by Collaborative Filtering", IEEE Trans. on Services Computing, 2011, vol.4, no.2, pp.140-152.

[4] L. Shao, J. Zhang, Y. Wei, J. Zhao, B. Xie, and H. Mei,'Personalized QoS prediction for Web services via collaborative filtering", in Proc. 5th International Conference on Web Services, 2007, pp. 439-446.

[5] M. Tang, Y. Jiang, J. Liu,X. F. Liu: "Location-Aware Collaborative Filtering for QoS-Based Service Recommendation", in Proc. 10th International Conference on Web Services, Hawaii, USA, June 2012, pp.202-209.

[6] G. Adomavicius, and A. Tuzhilin, Toward the next generation of recommender systems: a survey of the state-of-the-art and possible extensions",IEEE Trans. Knowledge and Data Engineering, 2005, pp.734 749.

[7] ]M. Alrifai, and T. Risse, "Combining Global Optimization with Local Selection for Efficient QoS-aware Service Composition", in Proc. of the International World Wide Web Conference, Apr. 2009, pp. 881-890.

[8] J. Wu, L. Chen, Y. Feng,Z. Zheng, M. Zhou, and Z. Wu, "Predicting QoS for Service Selection by Neighborhood-based Collaborative Filtering",IEEE Trans. on System, Man, and Cybernetics, Part A, 2013, vol. 43,no. 2, pp. 428-439.

[9] K. Elgazzar, R. Bell, and C. Volinsky,"Matrix factorization techniques for recommender systems",IEEE Computer, vol. 42 , no. 8, pp. 30-37,2009.

[10] L. Shao, J. Zhang, Y. Wei, J. Zhao, B. Xie, and H. Mei,'Personalized QoS prediction for Web services via collaborative filtering", in Proc. 5th International Conference on Web Services, 2007, pp. 439-446. 